\title{
Novel Active Light-emitting Traffic Signs Based on Backlight and Light Uniformity Technology
}

\author{
De-Kai ZHOU ${ }^{1, a}$, Jian-Xiao MA ${ }^{1, b,{ }^{*}}$, Chun-Hua TAN ${ }^{2, c}$, Ya-Yun ZHU ${ }^{1, d}$, \\ Gan LIU ${ }^{3, e}$ \\ ${ }^{1}$ Nanjing Forestry University, Nanjing, Jiangsu 210037, China \\ ${ }^{2}$ Baotou Landscape Company, Baotou, Neimenggu 014000,China \\ ${ }^{3}$ Nanjing SKY Traffic Safety Technology Stock CO., LTD Nanjing, Jiangsu 210037, China \\ azhoudekai@vip.qq.com, bjxma@163.com, ybgy3323778@163.com, d531411012@qq.com, \\ e269017285@qq.com \\ ${ }^{*}$ Corresponding author
}

Keywords: Backlight, Active Light-emitting, Traffic Signs, Light-Emitting Diode.

\begin{abstract}
Backlight and light uniformity technology are two key technologies for backlight module design of LCD. On the basis of discussing the advantages and disadvantages of the Intercalated LED traffic signs, backlight and light uniformity technology are creatively applied to the active light-emitting traffic signs in this paper. The novel traffic signs overcome the shortcomings of Intercalated LED traffic signs which follow solar road traffic signs (GA/T 580-2005), make the light-emitting mode of active light-emitting traffic signs upgrade from point emitting to surface emitting, so as to make the large guide signs to realize active light-emitting.
\end{abstract}

\section{Introduction}

Traffic signs are part of road traffic system. They play an important role in the normal operation of the road traffic. They convey information to road users based on color, shape, characters, graphics, such as facilities for traffic management [1].

With the development of society and economy, people put forward new requirements for landscape function, dealing with bad weather, taking care of vulnerable groups and other aspects except traffic function. Traffic signs can give people the sense of beauty, can transmit information not only to drivers but also to other road users, and can cope with frequent extreme weather conditions such as fog.

Now the active light-emitting technology used in prohibition signs, warning signs, mandatory signs and other small traffic signs is relatively mature. But there are some difficulties in applying it to large guide signs. On the basis of discussing the advantages and disadvantages of the Intercalated LED traffic signs, backlight and light uniformity technology are creatively applied to the active light-emitting traffic signs in this paper. And the paper invents a novel active light-emitting traffic signs based on backlight and light uniformity technology, so that large guide signs can be active light-emitting.

\section{Advantages and Disadvantages of the Intercalated LED Traffic Signs}

The traditional traffic signs depend on principle of reflection to transit information. When light irradiates on the traffic sign board made of reflective film, it would be reflected to the people's eyes by reflective film, so that people receive the information. The traditional traffic signs in order to realize information transmission need to have a combination of the following conditions: with an external light source, reflective film on the board has a good reflection performance and good visibility. That is, if any of these three conditions can't be met, the road users cannot obtain necessary traffic information and traffic safety problems would be arising therefrom.

With public safety industry standard solar road traffic signs (GA/T580-2005) promulgated, active light-emitting traffic signs like mushrooms are widely used all over the country. Active light-emitting traffic sign combines reflection and self light-emitting. When the environment bright enough it likes 
traditional traffic sign through reflection of reflective film to transmit information otherwise the LEDs would be lit to enhance visibility of the sign, so at night. LEDs are inserted in the plate so these signs are called Intercalated LED traffic signs in the paper. When the power is switch on, these light spots become lines and depict the outline of graph and text strokes, as shown in Figure 1.

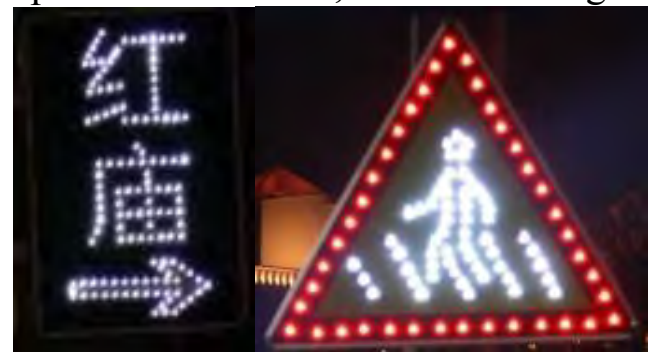

Fig. 1 Intercalated LED Traffic Signs

\section{Advantages of the Intercalated LED Traffic Signs}

Compared with the traditional traffic sign Intercalated LED traffic sign has the following advantages.

Adopting Reflective Films Combined with LEDs. The plate of Intercalated LED traffic sign is drilled at fixed interval according to the graphic outlines or text strokes what made of reflective films. Then LEDs are inserted into the holes and fixed on the plate. When environment is dark or at night LEDs would be lit and the traffic sign begin light-emitting. On the contrary, LEDs will be turn off and the information would be transferred by reflective films. Additionally, it also can work normally when circuit malfunction.

Active Light-emitting, Enhancing Distinguishing Distance. The use of LED has changed the history that the traffic sign wants to be noticed must depend on an external light source. And it can serve all road users [2]. Because of highlighted LEDs and special optical lens, the traffic sign has the strong permeability and good weather ability. The distinguishing distance of intercalated LED traffic sign is twice as long as that of conventional sign at night and four times in bad weather conditions, so it can effectively deal with adverse weather conditions such as fog and haze.

Take Solar as the Energy. Because of using solar energy the sign can work around the clock wherever it is [3]. Solar is clean, low carbon, green and tallies with the concept of sustainable development.

\section{Disadvantages of the Intercalated LED Traffic Signs}

Although the intercalated LED traffic signs have those advantages, there are still many shortcomings:

Undermined the Integrity of Reflective Films. LEDs are intercalated in the plate so the reflective films and plate must be drilled that would undermine the integrity of reflective films and greatly reduce the reflection coefficient. When circuit malfunction and LEDs would not be lit, the function of the traffic signs would be weakened.

Pixel Pitch Greatly Effect on the Display Performance. Intercalated LED traffic signs need to consider the size of the pixel pitch. Different pixel pitch will produce a different display performance, as shown in Figure 2. If it is too wide the performance of graphic or text consisted of the light-emitting body would be weakened. On the contrary the resolution of graphic or text will be increased but the production cost will be increased too.
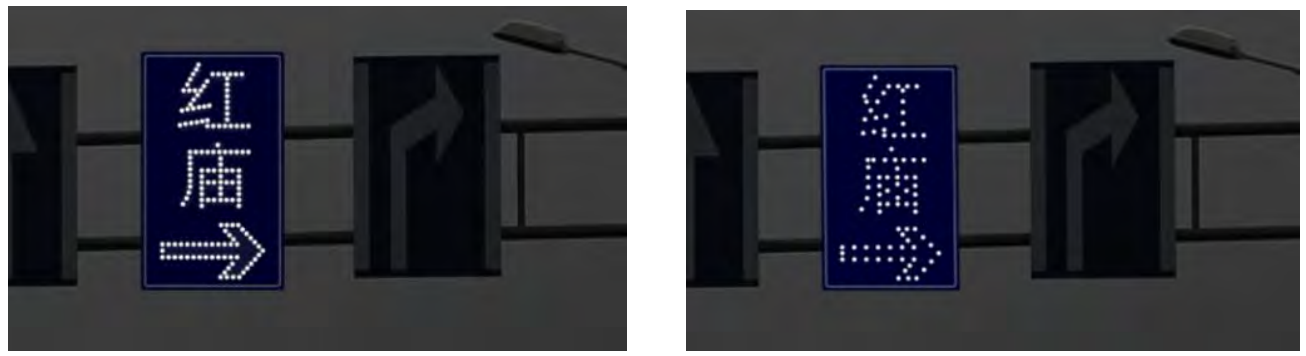

Fig. 2 A Contrast of Different Pixel Pitches 
When a Few LEDs Fail, the Display Performance Will Be Weakened. Even the meaning of the sign would be completely changed. As shown in Figure 3.
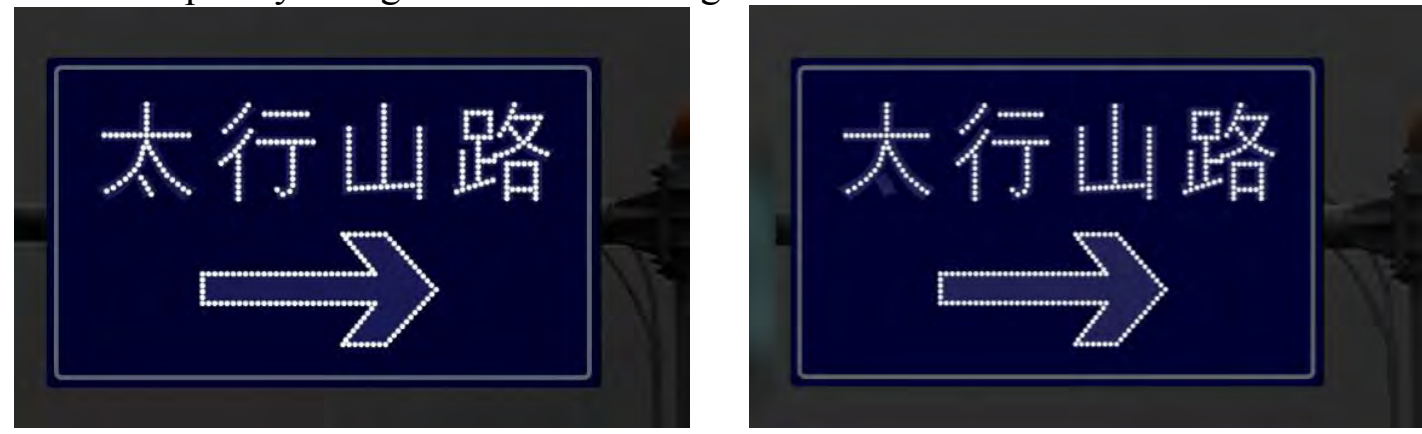

Fig. 3 A Contrast of the Display when LEDs Are All Normal or Some Are Abnormal

Difficult to Apply to Large Guide Signs which Have Many Texts. Intercalated LED traffic signs adopt such a light mode: several LEDs constitute lines and the lines depict the outline of graphs and text strokes. When graphic profile is complex or text strokes are more this traffic sign would be difficult to perform competently.

\section{Novel Traffic Signs based on Backlight and Light Uniformity Technology}

\section{Backlight and Light Uniformity Technology}

Backlight and light uniformity technology was first applied to liquid crystal display (LCD). Backlight is a kind of light source located on the back of the LCD. LCD does not shine, it displays graphics or characters because of backlight[4]. Light uniformity technology is a key technology for converting point light-emitting to surface light-emitting. The most typical application of light uniformity technology for LCD is light guide plate (LGP). Converting a point light source or a linear light source into the surface optical field distribution is the main function of the LGP. That is to say the light focused on the edge or at the back of LGP could be uniformly distributed on the output face[5].

Compared with Intercalated LED traffic signs, novel active light-emitting traffic signs make LEDs be transferred from the surface to inside. So the paper calls these signs as Inside LED traffic signs.

\section{Structural Design for Inside LED Traffic Signs}

The key of structural design for Inside LED traffic signs is that how to make point light source to be surface light source and transmit through the plate and the reflective films.

The structural of novel sign is similar to Intercalated LED traffic sign: the box structure, the thickness of $40 \mathrm{~mm} \sim 50 \mathrm{~mm}$, made of aluminum alloy profile frame, aluminum alloy plate for box panel. Different is that the graphics or characters of Inside traffic sign are carved into hollow instead of drilling, as shown in Figure 4. Considering the ability to resist wind the size and spacing of the connecting bars must be designed. If the width of connecting bars is $5 \mathrm{~mm}$ and the spacing is $50 \mathrm{~mm}$, deformation of the sign under $40 \mathrm{~m} / \mathrm{s}$ wind is not more than $8 \mathrm{~mm}$.

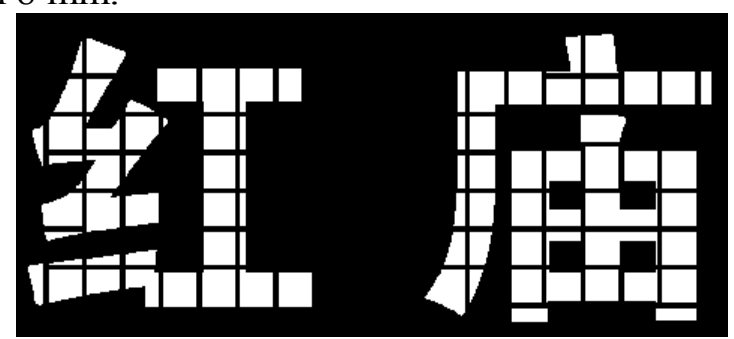

Fig. 4 Hollow Structure of Faceplate

LED light source module is fixed on the baseplate. In order to ensure light emitted from the light emitting surface be uniformity and soft, a LGP is set under the faceplate which can make the light be uniformity. 
Figure 5 shows the hierarchy of Inside LED traffic signs.

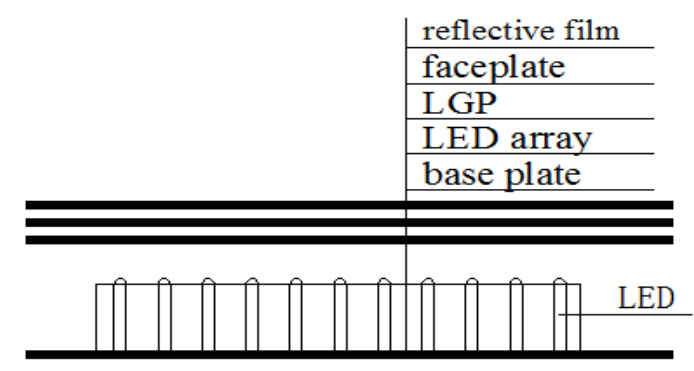

Fig. 5 The Hierarchy of Inside LED Traffic Signs

\section{Backlight Module Design for Inside LED Traffic Signs}

The design for light is to determine the number of LEDs and row pitch of LED matrix.

There is a close relationship between the distinguishing distance of active light-emitting sign and the brightness. Different distinguishing distance corresponds to different brightness. Assume that the brightness for $200 \mathrm{~m}$ is $\mathrm{L}_{200}$, according to transmittance of the reflective film the brightness of light out from LGP can be determined:

$$
\mathrm{L}_{\text {film }}=\mathrm{L}_{200} / \eta_{\text {film }}
$$

Where: $\mathrm{L}_{\text {film }}$ is the brightness of light out from LGP; $\eta_{\text {film }}$ is the transmittance of the reflective film. Similarly we can draw the brightness of light entering LGP:

$$
\mathrm{L}_{\mathrm{LGP}}=\mathrm{L}_{\text {film }} / \eta_{\mathrm{LGP}}
$$

Where: $\mathrm{L}_{\mathrm{LGP}}$ is the brightness of light entering LGP; $\eta_{\mathrm{LGP}}$ is the transmittance of the LGP.

Then according to the optical parameters and light use efficiency of the LED, we can calculate how many LED should be used.

$$
\mathrm{n}=\mathrm{L}_{\mathrm{LGP}} / \eta_{\mathrm{LED}}
$$

Where: $\mathrm{n}$ is the number of LED; $\eta_{\text {LED }}$ is light use efficiency of the LED. The size of a single LED matrix is equal to the size of the character and the row spacing is equal to the column spacing too in the paper. So the row pitch of LED matrix can be determined.

Figure 6 is a photo of new novel active light-emitting traffic sign applied to the second phase of Lukou International Airport.

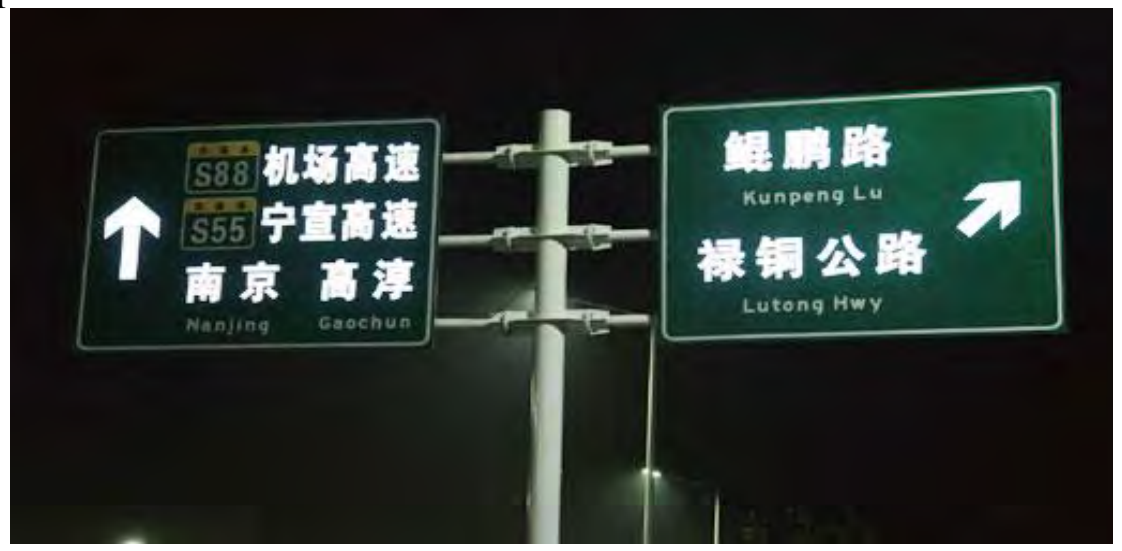

Fig. 6 An Example of Application of the New Novel Traffic Sign.

\section{Advantages and Disadvantages of the Inside LED Traffic Signs}

Obviously, as an active light-emitting traffic sign, Inside LED traffic sign has the advantage of the Intercalated LED traffic sign. Of course, as a new product it also has its own unique advantages and disadvantages. 


\section{Advantages of the Inside LED Traffic Signs}

Ensure the Integrity of Reflective Films. Because the LEDs are set in the sign, there is no need to drill on the reflective films so as to ensure the integrity of reflective films and make the sign work normally when it is unlighted.

Surface Light-emitting Mode Becomes True. Inside LED traffic signs make the point light source be converted to surface light source. Because the graphics or characters are no longer formed by LEDs, even if one or several LEDs go wrong the function of signs will not be affected.

Because of light uniformity technology the luminous surface is more uniform, softer. Compared with Intercalated LED traffic signs, novel signs can overcome the shortcomings, such as dazzling and hyphenation problem.

Modular Design Makes Installation and Maintenance to be Easy. Inside LED traffic signs make the light source be independent modules so that it is no longer bound by the text strokes and the light modules can be mass produced separately. When the display of signs becomes poor for some failed LEDs the light modules can be easily replaced, but Intercalated LED traffic signs must pull out the failed LEDs and then insert the new LEDs, so the plate and reflective films may be easily damaged during maintenance.

\section{Disadvantages of the Inside LED Traffic Signs}

Cannot Use Solar Because of Larger Power for the Present. Now Inside LED traffic signs are large guide signs, so the power of backlight module is large than Intercalated LED traffic signs. According to current engineering practice the signs cannot use solar, so the location where the signs are set must be able to provide commercial power.

Not All Information can be Luminous. Limited to the requirements of structural mechanics not all the information of the plate can be carved into hollow, such as English, mileage and other smaller characters.

\section{Conclusion}

Active light-emitting traffic signs get rid of the constraint of external light source so that the signs can take care of all the road users and can cope with a severe climate conditions and make the traffic signs be a part of the urban landscape. They will be the development trend of traffic signs. The novel active light-emitting traffic signs based on backlight and light uniformity technology change the existing light-emitting mode and make the mode upgrade from point emitting to surface emitting, so that active light-emitting technology can be applied to the large guide signs which have more characters than small signs. It is a great progress of active light-emitting traffic signs industry.

\section{References}

[1]Research Institute of Highway Ministry of Transport, Road traffic signs and markings - Part 2: Road traffic signs, Standards Press of China, Beijing, china, 2009.

[2]Nima Zhuoma, The Study of Method of Active Luminous Induction Facility Safety Based on the Visual Effect, Road Traffic Technology. 84(2011)7-9.

[3]Zhang Jianzhong, The Application of Sloar LED Road Traffic Signs to Trunk Highway Reconstruction, Shanxi Science \& Technology of Communications. 212(2011) 78-80.

[4]Zou Yuejun, Structure analysis of LCD Backlight and Some Methods for Improving its Luminance, Chinese Journal of Liquid Crystals and Displays. 17(2002)465-470.

[5]Chen Xiangxian, Xu Ping, Wan Lili, Huang Jiefeng, Novel Technologies of Light Guide Plate in Backlight System. 22(2006)576-582. 